11

\title{
Хаотический генератор с выделенной инерционностью высокой мощности на биполярном транзисторе
}

\author{
() С.В. Савельев, Л.А. Морозова \\ Фрязинский фрилиал Института радиотехники и электроники им. В.А. Котельникова РАН, \\ 141190 Фрязино, Московская обл., Россия \\ e-mail: mila-morozova.ludmila@yandex.ru
}

Поступило в Редакцию 19 декабря 2019 г.

В окончательной редакции 15 мая 2020 г.

Принято к публикации 15 мая 2020 г.

Впервые изложены результаты исследования возможности создания мощного широкополосного источника хаотического сигнала микроволнового диапазона длин волн на основе однотранзисторной системы на мощном биполярном транзисторе путем реализации генератора с выделенной инерционностью. Произведены теоретические расчеты, показывающие возможность построения такого генератора. Разработан экспериментальный макет генератора хаоса с выделенной инерционностью в гибридно-интегральном исполнении на основе отечественного мощного транзистора 2Т982A-2. Продемонстрирована возможность генерации хаотического СВЧ-сигнала с центральной частотой $4.55 \mathrm{GHz}$ и интегральной мощностью $1.1 \mathrm{~W}$. Эффективная ширина спектра мощности хаотического сигнала составляла $11 \%$ при неравномерности спектральной характеристики $3 \mathrm{~dB}$, спектральная плотность генерируемых шумовых колебаний была $2.2 \cdot 10^{-3} \mathrm{~W} / \mathrm{MHz}$, КПД равнялся $15 \%$.

Ключевые слова: мощный биполярный транзистор, динамический хаос, генератор с выделенной инерционностью.

DOI: 10.21883/JTF.2020.12.50135.418-19

\section{Введение}

Генерирование шумовых широкополосных сигналов микроволнового диапазона с высоким энергетическим потенциалом тесно связано с созданием систем генерации на мощных транзисторах [1-4]. Так, в [1] предложена модель микроволнового широкополосного генератора на мощном биполярном транзисторе, найдены значения параметров модели, отвечающие генерации хаоса, экспериментально показано соответствие теоретической модели реальному генератору на мощном биполярном транзисторе. В работе [2] представлен широкополосный генератор хаотического сигнала микроволнового диапазона на мощном отечественном транзисторе 2Т982А-2 с $3.4 \%$-й полосой генерируемых колебаний, центральной частотой $5.35 \mathrm{GHz}$, интегральной мощностью $230 \mathrm{~mW}$, неравномерностью огибающей спектра мощности, не превышающей $3 \mathrm{~dB}$, и КПД 10\%. Относительно невысокое значение КПД генератора определялось пониженным значением напряжения питания транзистора относительно номинального для выхода в режим генерации хаотических колебаний. В [3] описан генератор хаоса на транзисторе такого же типа, содержащий дополнительный нелинейный контур, в котором роль нелинейного элемента играет варакторный диод. Генератор хаотического СВЧ-сигнала имел эффективную полосу колебаний 10\% при интегральной мощности до $600 \mathrm{~mW}$ и КПД 20-25\%. Интересный подход по созданию транзисторных генераторов хаоса предложен в [4], где в качестве нелинейного элемента использована спин-волновая линия передачи в кольцевой автоколеба- тельной системе на полевых транзисторах с возможностью оперативного управления хаотической динамикой системы с помощью внешнего СВЧ воздействия [5]. Интегральная мощность хаотического сигнала на выходе такого генератора составляла $637 \mathrm{~mW}$ при полосе частот $943 \mathrm{MHz}$ с центральной частотой $2.95 \mathrm{GHz}$ и КПД до 7\%. Однако неравномерность огибающей спектра мощности хаотического сигнала превышала $15 \mathrm{~dB}$, что ограничивает область применения таких генераторов.

Рассмотренные системы генерирования хаоса микроволнового диапазона являются, в соответствии с литературными источниками, практически всем известными транзисторными источниками шумовых колебаний с большим энергетическим потенциалом со спектральной плотностью $10^{-3} \mathrm{~W} / \mathrm{MHz}$ по порядку величины. Однако их практическое применение ограничивает сильная зависимость режима генерации хаоса от температуры, значений напряжений питания, времени эксплуатации. Кроме того, эти приборы требуют сложной настройки при изготовлении.

В настоящей работе для моделирования микроволновой хаотической системы использовался генератор с выделенной инерционностью, впервые введенный в рассмотрение в [6]. Модель детально изучена в работах $[1,2,7]$, определено множество параметров, отвечающих различным типам колебательного процесса системы. Установлено, что модель генератора с выделенной инерционностью адекватно описывает процессы в системах на мощных биполярных транзисторах.

Целью настоящей работы является создание микроволновой системы генерации хаоса на мощном биполяр- 
ном транзисторе в соответствии со схемой генератора с выделенной инерционностью.

\section{1. Теоретическая часть}

Генератор с выделенной инерционностью представляет собой RLC-генератор с индуктивной обратной связью $[1,6]$ и инерционным преобразованием выходного сигнала нелинейного усилителя. Динамическая характеристика усилительного элемента генератора имеет линейный участок и участок с насыщением. Инерционный модуль, преобразующий выходной сигнал усилительного элемента, собран по схеме однополупериодного квадратичного детектора. Основным параметром, определяющим динамику генератора, является параметр инерционности, определяемый как $g=\left(f \tau_{f}\right)^{-1}$, где $f-$ характеристическая частота RLC-генератора, определяемая сопротивлением и емкостью входного контура нелинейного усилителя, $\tau_{f}=R_{f} C_{f}-$ постоянная времени инерционного преобразователя, $R_{f}$ и $C_{f}-$ значения сопротивления и емкости фильтра цепи инерционного преобразователя. В $[1,6,7]$ было установлено, что система переходит в режим развитого хаоса при значениях параметра инерционности:

$$
g_{K} \leq 0.06
$$

В настоящей работе для реализации модели генератора с выделенной инерционностью в СВЧ-диапазоне будем исходить из того, что крутизна $\mathrm{S}$ динамической характеристики мощного транзистора в первом приближении прямо пропорциональна значению напряжения питания [8]

$$
S=r\left(U_{0}-U_{p}\right),
$$

где $U_{0}-$ постоянная и $U_{p}-$ изменяющаяся во времени составляющие напряжения питания, $r-$ коэффициент. Положим, что изменяющаяся во времени составляющая напряжения питания $U_{p}$ суть напряжение с выхода инерционного преобразователя. Тогда, согласно математической модели генератора с выделенной инерционностью [7]:

$$
r=\frac{b}{\omega_{0}^{2} M},
$$

где $b$ - параметр, определяющий глубину модуляции крутизны динамической характеристики, $\omega_{0}$ - круговая частота, $M$ - параметр, определяющий величину положительной обратной связи. Для крутизны линейного участка динамической характеристики справедливо

$$
S=S_{0}-\frac{b}{\omega_{0}^{2} M} U_{p},
$$

где $S_{0}=r U_{0}-$ постоянная составляющая крутизны динамической характеристики.

Для напряжения на входе нелинейного усилителя $\Psi$ в системе генератора с выделенной инерционностью на линейном участке динамической характеристики, когда
$\Psi \leq k$, где $k-$ предельное значение напряжения линейного участка, справедливо:

$$
\ddot{\Psi}+\omega_{0}^{2} \Psi=\left(\mu-b U_{p}\right) \dot{\Psi}-b \dot{U}_{p} \Psi,
$$

где $\mu=\omega_{0} M S_{0}-R / L, R-$ сопротивление во входном контуре нелинейного усилителя, $L-$ индуктивность в цепи обратной связи. Переходя к безразмерным переменным $\tau=\omega_{0} t, X=\Psi\left(b / \omega_{0}\right)^{1 / 2}, Z=U_{P} b / \omega_{0}$, $Y=\Omega\left(b / \omega_{0}^{3}\right)^{1 / 2}$, где $\Omega$ - ток в цепи положительной обратной связи генератора, $t-$ время и, учитывая, что $\dot{\Omega}=-\omega_{0}^{2} \Psi$, уравнение (8) можно переписать как

$$
\begin{gathered}
\dot{X}=Y+\left(m_{1}-m_{2}\right) X-Z X, \\
\dot{Y}=-X,
\end{gathered}
$$

где $m_{1}=M \omega_{0} S_{0}, m_{2}=R(C / L)^{1 / 2}$ - параметры возбуждения и диссипации соответственно. Следуя [7], для участка с насыщением, $\Psi>k$, можно записать

$$
\begin{gathered}
\dot{X}=Y-m_{2} X-q Z, \\
\dot{Y}=-X,
\end{gathered}
$$

где безразмерный параметр ограничения $q=k\left(b / \omega_{0}\right)^{1 / 2}$.

Связь между входным $U$ и выходным $U_{P}$ напряжениями однополупериодного инерционного преобразователя в соответствии с [7] представима как

$$
\dot{U}_{p}=-g U_{p}+g F(U) U^{2}, \quad F(U)= \begin{cases}1, & U \geq 0, \\ 0, & U<0,\end{cases}
$$

где $F(U)$ - единичная функция Хевисайда. Для напряжения $U$ справедливо

$$
U=L_{1} \dot{\Omega}-M \dot{I}
$$

где $I$ - ток во входном контуре нелинейного усилителя, $L_{1}$ - индуктивность в цепи обратной связи. Подстановка (9) в (8) с учетом соотношений, полученных в [8], при введении безразмерной переменной $W=I\left(b / \omega_{0}^{3}\right)^{1 / 2}$ и при условии $L_{1}=L=M$, дает выражения

$$
\begin{gathered}
\dot{Z}=-g Z+s F\left(2 X-m_{2} W\right)\left(2 X-m_{2} W\right)^{2}, \\
\dot{W}=X-m_{2} W,
\end{gathered}
$$

где $g=R C / \tau_{f}$ - параметр инерционности, $R$ и $C-$ сопротивление и емкость входного контура нелинейного усилителя. Тогда, используя (7), (8) и (10), уравнения, описывающие динамику генератора с инерционной модуляцией напряжения питания активного элемента, можно представить в виде четырехмерной четырехпараметрической нелинейной диссипативной системы:

$$
\begin{gathered}
\dot{X}=Y+\left(m_{1}-m_{2}\right) X-X Z, \quad X \leq q, \\
\dot{X}=Y-m_{2} X-q Z, \quad X>q, \\
\dot{Y}=-X, \\
\dot{Z}=-g Z+g F\left(2 X-m_{2} W\right)\left(2 X-m_{2} W\right)^{2}, \\
\dot{W}=X-m_{2} W,
\end{gathered}
$$



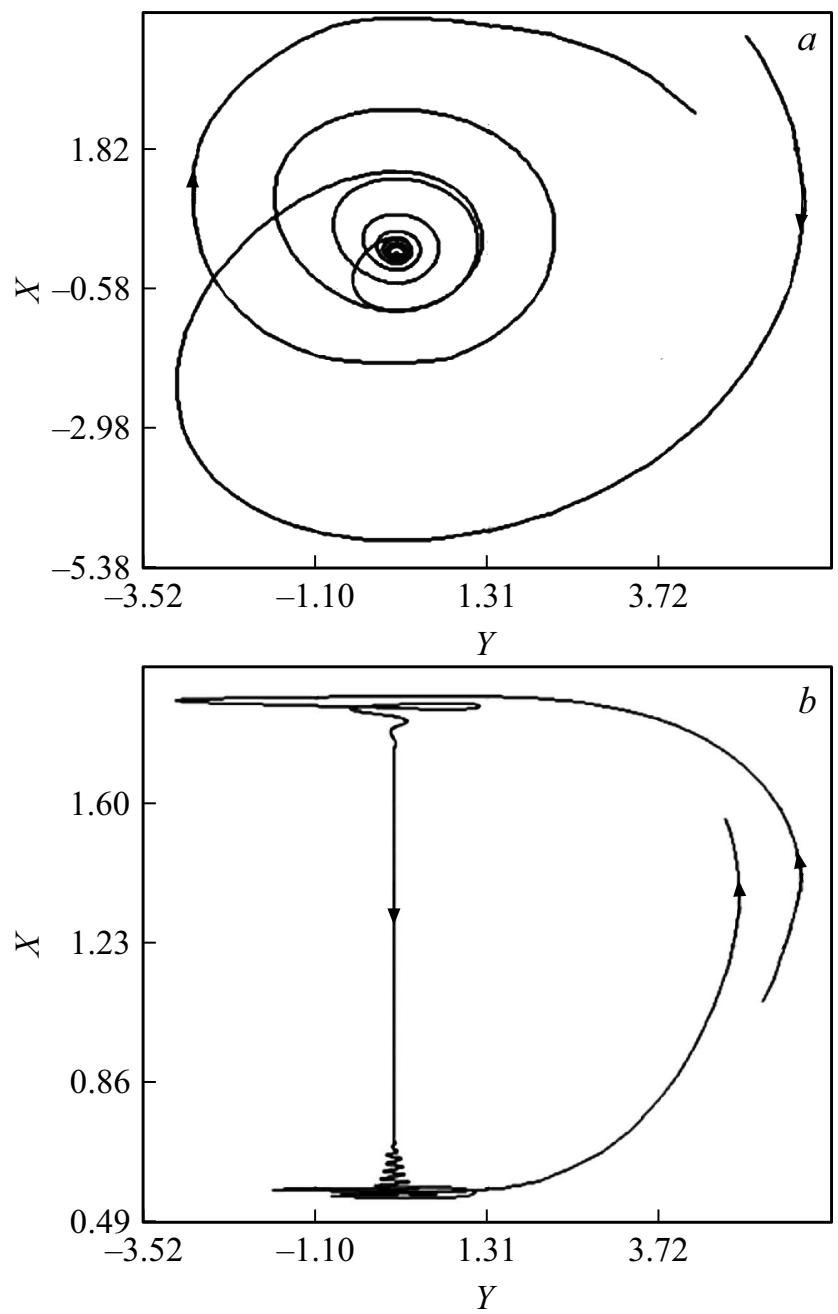

Рис. 1. Проекция движения изображающей точки системы (11) на плоскость $X, Y(a)$ и на плоскость $X, Z(b)$.

Полученная система (11) равнозначна математической модели генератора с выделенной инерционностью [7]. Динамика модели определяется двумя механизмами ограничения колебаний. Первый механизм безынерционный и связан с нелинейностью характеристики усилительного элемента. Второй - инерционный, обусловлен влиянием напряжения с выхода инерционного преобразования на крутизну нелинейного усилителя. Основным параметром, определяющим вид колебательного процесса в исследуемой системе, является параметр инерционности $g$. При уменьшении параметра инерционности от значения, равного единице, в системе наблюдаются бифуркации периода колебаний от однотактного устойчивого цикла с последовательным увеличением периода колебательного процесса на единицу. При этом зоны регулярных колебаний разделены зонами хаоса на основе механизма перемежаемости со случайным распределением цугов колебаний с соседними периодами. При каждой последующей бифуркации расстояние между бифуркационными значениями параметра инерционности уменьшаются, зоны хаотических колебаний располагаются плотнее. Характер развития колебаний в исследуемой системе позволяет рассчитывать на существование сходимости последовательности бифуркаций с увеличением кратности устойчивых циклов системы на единицу, что позволяет определить точку сгущения последовательности бифуркационных значений параметра инерционности: $g_{k}=0.06$. При значениях $g \leq g_{k}$ система находится в зоне развитого хаоса, где анализ временных реализаций показывает, что система демонстрирует последовательность цугов колебаний с произвольным, но близким по значению к $g$, количеством единичных колебаний характеристической частоты системы и случайной начальной фазой. На рис. 1 представлены фазовые портреты системы (11) в двух плоскостях, полученные для $m_{1}=1.6, m_{2}=0.2$, $q=1, g=0.05$. В начальный момент времени амплитуда колебаний медленно растет, достигая максимума, затем происходит срыв колебаний и возвращение системы в область начальной изображающей точки. В фазовом пространстве системы реализуется странный аттрактор типа аттрактора Шильникова [9]. Наличие аттрактора Шильникова и перемежаемость указывает на развитую хаотическую динамику системы (11).

Полученные результаты показывают, что для построения экспериментального макета генератора с выделенной инерционностью в СВЧ-диапазоне необходимо соблюдение условия (1), накладываемое на значение параметра инерционности.

\section{2. Эксперимент}

Необходимым и достаточным условием генерации хаотических колебаний в однотранзисторной системе с выделенной инерционностью является введение цепи однополупериодного инерционного преобразования выходного сигнала активного элемента при выполнении условия (1). При этом активный элемент может работать в штатном режиме, т.е. значения напряжений питания могут быть близки максимальным паспортным, что позволяет получить мощный хаотический сигнал на выходе генератора. Экспериментальный макет генератора хаоса был изготовлен по гибридно-интегральной микрополосковой технологии на материале ФЛАН 10 толщиной $1 \mathrm{~mm}$. В состав генератора входит (рис. 2) транзистор 2Т982А-2, предназначенный для работы в схемах с общей базой, эмиттер и коллектор которого подсоединены к согласующим площадкам микрополосковых линий. Питание транзистора осуществляется через микрополосковые фильтры на четвертьволновых шлейфах, настроенные на центральную частоту генератора, равную $4.55 \mathrm{GHz}$. Положительная обратная связь в системе осуществляется путем межвыводных емкостей транзистора. Такая транзисторная система переходила в режим автогенерации при достижении напряжения на эмиттере транзистора, равного $-0.8 \mathrm{~V}$. В экспериментальном макете напряжения питания генератора составляли $-1.0 \mathrm{~V}$ на эмиттере и $+17 \mathrm{~V}$ на коллекторе. 
Основная часть мощности хаотического сигнала с выхода генератора поступала в нагрузку. 20\% мощности ответвлялось в цепь однополупериодного квадратичного детектора, включавшего в себя диод $D$ типа Д403В, производивший однополупериодное преобразование сигнала, и RC-фильтр с параметрами, удовлетворяющими условию $g=0.05$ для центральной частоты генератора. Значения элементов фильтра были следующие: $R=200 . \Omega, C=250 \mathrm{pF}$. Напряжение с выхода инерционного преобразователя подавалось на четвертьволновой шлейф цепи питания эмиттера.

Генерация хаоса в системе соответствовала колебаниям системы (11). При подаче напряжений питания происходило автовозбуждение колебаний в генераторе. При этом начинался рост положительного напряжения на выходе однополупериодного нелинейного преобразователя. Когда результирующее напряжение на эмиттере становилось более $0.8 \mathrm{~V}$, процесс генерации обрывался. Таким образом, как и в численном эксперименте, хаотический сигнал на выходе генератора представлял собой последовательность неповторяющихся цугов колебаний с произвольным числом единичных колебаний и случайной начальной фазой.

Спектр мощности и распределение плотности вероятности выходного сигнала генератора хаоса представлен на рис. 3. Эффективная полоса частот генерируемых

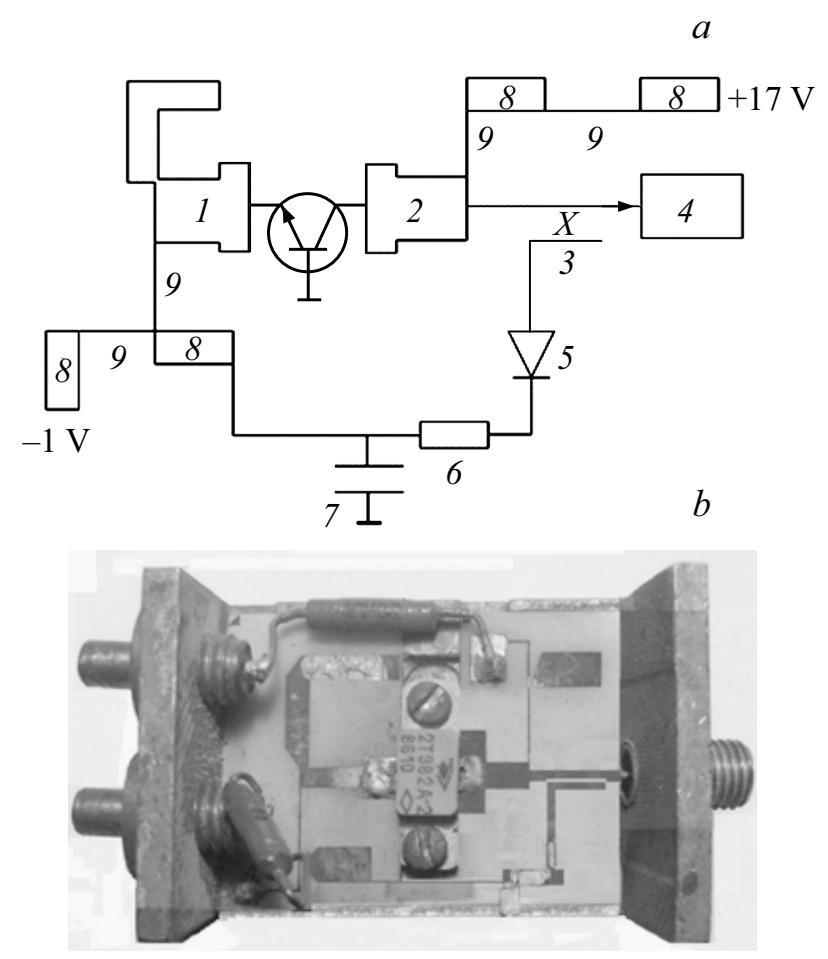

Рис. 2. Генератор с выделенной инерционностью в микроволновом диапазоне: $a-$ функциональная схема, $b-$ фотография макета. 1 - согласующая цепь эмиттера, $2-$ согласующая цепь коллектора, 3 - ответвитель электромагнитной мощности, 4 - нагрузка, 5 - диод Д403В, $6-$ сопротивление, 7 - конденсатор, 8 и $9-$ элементы фильтров питания на четвертьволновых линиях.
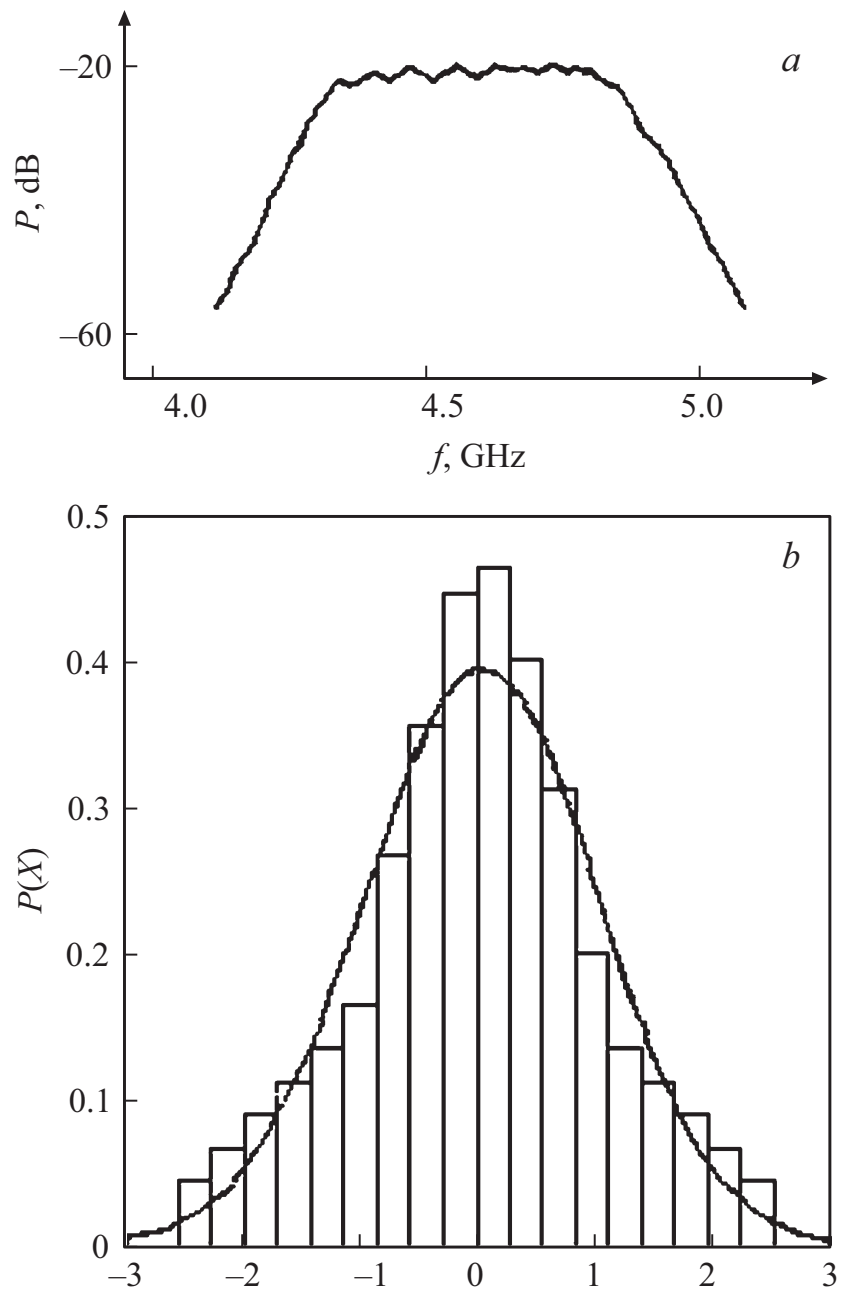

Рис. 3. Спектр мощности $(a)$ и распределение плотности вероятности $(b)$ хаотического сигнала генератора.

шумовых колебаний при неравномерности спектра мощности $3 \mathrm{~dB}$ занимала диапазон частот от 4.3 до $4.8 \mathrm{GHz}$, интегральная мощность сигнала составляла $1.1 \mathrm{~W}$, что для усредненной спектральной плотности генерируемых шумовых колебаний давало значение $2.2 \cdot 10^{-3} \mathrm{~W} / \mathrm{MHz}$. КПД генератора при рабочем токе транзистора $400 \mathrm{~mA}$ равнялся $15 \%$, распределение плотности вероятности близко к нормальному Гауссовому с положительным эксцессом 1.12.

\section{Заключение}

В работе предложен и реализован на практике генератор хаотических колебаний с выделенной инерционностью в микроволновом диапазоне длин волн на базе отечественного мощного транзистора 2Т982А-2. Генератор прост в исполнении и практически не требует настройки. Значительное, до $25 \%$, уменьшение коллекторного напряжения питания не приводило к срыву хаотических колебаний, при этом уменьшалась, до 50\%, интегральная мощность на выходе генератора. Режим 
генерации хаоса устойчив и к изменению температуры основания корпуса генератора, на который крепится база транзистора. Генерация хаотических колебаний сохранялась в диапазоне температур от -20 до $+80^{\circ} \mathrm{C}$. Устойчивость к внешним воздействиям совместно с параметрами выходного хаотического сигнала генератора позволяет рекомендовать его для использования в аппаратурах радиопротиводействия и активной шумовой радиолокации.

В заключение стоит отметить, что проведенные в работе теоретические и экспериментальные исследования генератора с выделенной инерционностью микроволнового диапазона длин волн позволяют предположить возможность его реализации на других типах активных элементов с зависимостью крутизны динамической характеристики от напряжения питания.

\section{Финансирование работы}

Работа выполнена по плану государственного задания.

\section{Конфликт интересов}

Авторы заявляют, что у них нет конфликта интересов.

\section{Список литературы}

[1] С.В. Савельев. РЭ, 49 (7), 850 (2004).

[2] С.В. Савельев. Письма в ЖТФ, 38 (11), 20 (2011).

[3] А.С. Дмитриев, Е.В. Ефремова, Н.А. Максимов, А.И. Панас. Генерация хаоса. (Техносфера, М., 2012) с. 359.

[4] С.В. Гришин, В.С. Гришин, Д.В. Романенко, Ю.П. Шараевский. Письма в ЖТФ, 40 (19), 51 (2014).

[5] S.V. Grishin, Yu.P. Sharaevskii, S.A. Nikitov, D.V. Romanenko. IEEE Trans. on Magnetics, 49 (3), 1047 (2013).

[6] С.В. Савельев. РЭ, 37 (6), 1064 (1992).

[7] С.В. Савельев. Журнал радиоэлектроники [электронный журнал], 6, (2017). Режим доступа: http://jre.cplire.ru/jre/jun17/10/text.pdf

[8] С. Зи. Физика полупроводниковых приборов (Мир, М., 1984) Книга 1. Изд. № 20/2918. Ч. 2, гл. 3, с. 190

[9] Л.П. Шильников. В сб. Проблемы нелинейных и турбулентных процессов в физике (Наук. думка, Киев, 1985) c. 118 . 